\title{
Analytical Results Connecting Stellar Structure Parameters and Extended Reaction Rates
}

\author{
Hans J. Haubold ${ }^{1,2}$ and Dilip Kumar ${ }^{2}$ \\ ${ }^{1}$ Office for Outer Space Affairs, United Nations, Vienna International Centre, P.O. Box 500, 1400 Vienna, Austria \\ ${ }^{2}$ Centre for Mathematical Sciences Pala Campus, Arunapuram P.O., Palai, Kerala 686 574, India
}

Correspondence should be addressed to Hans J. Haubold; hans.haubold@gmail.com

Received 5 March 2014; Accepted 6 April 2014; Published 12 May 2014

Academic Editor: Milan S. Dimitrijevic

Copyright (C) 2014 H. J. Haubold and D. Kumar. This is an open access article distributed under the Creative Commons Attribution License, which permits unrestricted use, distribution, and reproduction in any medium, provided the original work is properly cited.

\begin{abstract}
Possible modification in the velocity distribution in the nonresonant reaction rates leads to an extended reaction rate probability integral. The closed form representation for these thermonuclear functions is used to obtain the stellar luminosity and neutrino emission rates. The composite parameter $\mathscr{C}$ that determines the standard nuclear reaction rate through the Maxwell-Boltzmann energy distribution is extended to $\mathscr{C}^{*}$ by the extended reaction rates through a more general distribution than the MaxwellBoltzmann distribution. The new distribution is obtained by the pathway model introduced by Mathai (2005). Simple analytic models considered by various authors are utilized for evaluating stellar luminosity and neutrino emission rates and are obtained in generalized special functions such as Meijer's $G$-function and Fox's $H$-function. The standard and extended nonresonant thermonuclear functions are compared by plotting them. Behaviour of the new energy distribution, which is more general than the Maxwell-Boltzmann, is also studied.
\end{abstract}

\section{Introduction}

The mystery behind the distant universe is explored so far by the understanding of the sun, the star near to us. It is the only star whose mass, radius, and luminosity are fairly accurately known. The structural change in the sun is due to the central thermonuclear reactor in it. Solar nuclear energy generation and solar neutrino emission are governed by chains of nuclear reactions in the gravitationally stabilized solar fusion reactor $[1,2]$. Qualitative calculations of specific reaction rates require a large amount of experimental inputs and theoretical assumptions. By using the theories from nuclear physics and kinetic theory of gases one can determine the reaction rate for low-energy nonresonant thermonuclear reactions in nondegenerate plasma [3]. The formalization of the calculation of the reaction rate of interacting articles under cosmological or stellar conditions was presented by many authors $[4,5]$. For the most common case, a nuclear reaction in which a particle of type 1 strikes a particle of type 2 producing a nucleus 3 and a new particle 4 is symbolically represented as

$$
1+2 \longrightarrow 3+4+E_{12}
$$

where $E_{12}$ is the energy release given by $E_{12}=\left(m_{1}+m_{2}-\right.$ $\left.m_{3}-m_{4}\right) c^{2}$, where $m_{i}, i=1,2,3,4$ denote the masses of the particles and $c$ denotes the velocity of light. The reaction rate $r_{12}$ of the interacting particles 1 and 2 is obtained by averaging the reaction cross section over the normalized density function of the relative velocity of the particles [5-7]. Let $n_{1}$ and $n_{2}$ denote the number densities of the particles 1 and 2 , respectively, and let $\sigma(v)$ be the reaction cross section where $v$ is the relative velocity of the particles and $f(v)$ is the normalized velocity density; then the reaction rate $r_{12}$ is given by

$$
\begin{aligned}
r_{12} & =\left(1-\frac{1}{2} \delta_{12}\right) n_{1} n_{2}\langle\sigma v\rangle_{12} \\
& =\left(1-\frac{1}{2} \delta_{12}\right) n_{1} n_{2} \int_{0}^{\infty} v \sigma(v) f(v) \mathrm{d} v \\
& =\left(1-\frac{1}{2} \delta_{12}\right) n_{1} n_{2} \int_{0}^{\infty} \sigma(E)\left(\frac{2 E}{\mu}\right)^{1 / 2} f(E) \mathrm{d} E
\end{aligned}
$$

where $\delta_{12}$ is the Kronecker delta which is introduced to avoid double counting in the reaction if particles 1 and 2 
are identical. $\langle\sigma v\rangle_{12}$ is the thermally averaged product which is in fact the probability per unit time that two particles 1 and 2 confined to a unit volume will react with each other. $\mu$ is the reduced mass of the particles given by $\mu=$ $\left(m_{1} m_{2}\right) /\left(m_{1}+m_{2}\right) . E=\mu v^{2} / 2$ is the kinetic energy of the particles in the centre of mass system. From literature $[4,5,7]$ it may be noted that all the analytic expressions for astrophysically relevant nuclear reaction rates underline the hypothesis that the distribution of the relative velocities of the reacting particles always remains Maxwell-Boltzmann for a nonrelativistic nondegenerate plasma of nuclei in thermodynamic equilibrium. The Maxwell-Boltzmann relative kinetic energy distribution can be written as

$$
f_{\mathrm{MBD}}(E) \mathrm{d} E=2 \pi\left(\frac{1}{\pi k T}\right)^{3 / 2} \exp \left(-\frac{E}{k T}\right) \sqrt{E} \mathrm{~d} E
$$

where $k$ is the Boltzmann constant and $T$ is the temperature. Substituting (3) in (2) we get

$$
\begin{aligned}
r_{12}= & \left(1-\frac{1}{2} \delta_{12}\right) n_{1} n_{2}\left(\frac{8}{\pi \mu}\right)^{1 / 2}\left(\frac{1}{k T}\right)^{3 / 2} \\
& \times \int_{0}^{\infty} E \sigma(E) \exp \left(-\frac{E}{k T}\right) \mathrm{d} E .
\end{aligned}
$$

The thermonuclear fusion depends on three physical variables, the temperature $T$, the Gamow energy $E_{G}$, and the nuclear fusion factor $S(E)$. If two nuclei of charges $Z_{1} e$ and $Z_{2}$ e collide at low energies below the Coulomb barrier, then the Gamow energy $E_{G}$ is given by $[8,9]$

$$
E_{G}=2 \mu\left(\pi \alpha Z_{1} Z_{2} c\right)^{2}
$$

where $\alpha$ is the electromagnetic fine structure constant given by

$$
\alpha=\frac{e^{2}}{\hbar c}
$$

where $e$ is the quantum of electric charge, $\hbar$ is Planck's quantum of action, and $\alpha$ is approximately $1 / 137$ [9] for our universe. Thus the Gamow factor, which is determined by the electromagnetic force, and the nuclear fusion factor $S(E)$ set the nuclear reaction cross section at low energies for nonresonant charged particles as $[7,10]$

$$
\sigma(E)=\frac{S(E)}{E} \exp \left[-\left(\frac{E_{G}}{E}\right)^{1 / 2}\right],
$$

and $S(E)$ is the cross section factor which is often found to be constant or a slowly varying function of energy over a limited range of energy given by $[4,5]$

$$
\begin{aligned}
S(E) & \approx S(0)+\frac{\mathrm{d} S(0)}{\mathrm{d} E} E+\frac{1}{2} \frac{\mathrm{d}^{2} S(0)}{\mathrm{d} E^{2}} E^{2} \\
& =\sum_{\nu=0}^{2} \frac{S^{(\nu)}(0)}{\nu !} E^{\nu} .
\end{aligned}
$$

Substituting (7) and (8) in (4) we obtain

$$
\begin{aligned}
r_{12}= & \left(1-\frac{1}{2} \delta_{12}\right) n_{1} n_{2}\left(\frac{8}{\pi \mu}\right)^{1 / 2} \\
& \times\left(\frac{1}{k T}\right)^{3 / 2} \sum_{\nu=0}^{2} \frac{S^{(\nu)}(0)}{\nu !} \\
& \times \int_{0}^{\infty} E^{\nu} \exp \left[-\frac{E}{k T}-\left(\frac{E_{G}}{E}\right)^{1 / 2}\right] \mathrm{d} E .
\end{aligned}
$$

This is the nonresonant reaction rate probability integral in the Maxwell-Boltzmann case. The closed form evaluation of this integral can be seen in a series of papers by Mathai and Haubold; see, for example, Haubold and Mathai $[6,11]$, Mathai and Haubold [5], and so forth. The main aim of the present work is to extend the reaction rate probability integral given in (9) by replacing the Maxwell-Boltzmann energy distribution by a more general energy distribution called the pathway energy distribution obtained by using the pathway model of Mathai introduced in 2005.

The paper is organized as follows. In the next section we discuss a more general energy distribution than the MaxwellBoltzmann distribution and obtain the extended reaction rate probability integral in the nonresonant case. We take advantage of the closed form representation of the extended thermonuclear reaction rate for finding the luminosity and the neutrino emission rate of the nonlinear stellar model under consideration in Section 3. Section 4 is devoted to finding the desired connection between stellar structure parameters and the neutrino emission of the stellar model by using the closed form analytic representation of the extended reaction rates. A comparison of the Maxwell-Boltzmann energy distribution with the pathway energy distribution is done with the help of graphs in Section 5. Also we try to discriminate the standard and extended reaction rates. Concluding remarks are included in Section 6.

\section{Extended Nonresonant Thermonuclear Reaction Rate and Its Closed Forms}

In recent years, possible deviations of the velocity distribution of the plasma particles from the Maxwell-Boltzmann in connection with the production of neutrinos in the gravitationally stabilized solar fusion reactor have been pointed out $[2,10,12-15]$. It was initiated by Tsallis, the originator of nonextensive statistical mechanics [16-18], who has used $q$ exponential function as the fundamental distribution instead of the Maxwell-Boltzmann distribution. An initial attempt to extend the standard theories of reaction rates to Tsallis statistics was done by many authors; see Mathai and Haubold [19] and Saxena et al. [20]. In 2005, Mathai introduced the pathway model by which even more general distributions can be incorporated in the theory of reaction rates $[19,21]$. Initially, pathway model was introduced for the matrix variate case to cover many of the matrix variate statistical densities. The scalar case is a particular one there. Later, Mathai, his coworkers, and others found connection of pathway model 
with the information theory, the fractional calculus, the Mittag-Leffler functions, and so forth. The pathway model can be effectively used in any situation in which we need to switch between three different functional forms, namely, generalized type- 1 beta form, generalized type- 2 beta form, and generalized gamma form, using the pathway parameter $q$. In practical purpose of fitting experimental data, pathway model can be utilized to switch between different parametric families with thicker or thinner tail. The pathway model for the real scalar case can be explained as follows:

$$
\begin{gathered}
f_{1}(x)=c_{1} x^{\gamma-1}\left[1-a(1-q) x^{\delta}\right]^{1 /(1-q)}, \\
a>0, \quad \delta>0, \quad 1-a(1-q) x^{\delta}>0, \quad \gamma>0, \quad q<1,
\end{gathered}
$$

is the generalized type-1 beta form of the pathway model. This is a model with right tail cut-off for $q<1$. The Tsallis statistics for $q<1$ can be obtained from this model by putting $\gamma=1$ [16-18]. Other cases available are the regular type-1 beta density, the Pareto density, the power function, and the triangular and related models [22]. The generalized type- 2 beta form of the pathway model is given by

$$
\begin{array}{r}
f_{2}(x)=c_{2} x^{\gamma-1}\left[1+a(q-1) x^{\delta}\right]^{-1 /(q-1)}, \\
0<x<\infty, \quad q>1, \quad a>0, \quad \gamma>0, \quad \delta>0 .
\end{array}
$$

Here also for $\gamma=1$ we get the Tsallis statistics for $q>1$ [1618]. Other standard distributions coming from this model are regular type- 2 beta density, $F$-distribution, the Lévy model, and related models [22]. When $q \rightarrow 1, f_{1}(x)$ and $f_{2}(x)$ will reduce to the generalized gamma form of the pathway model given by

$$
f_{3}(x)=c_{3} x^{\gamma-1} \mathrm{e}^{-a x^{\delta}}, \quad x>0
$$

This model covers generalized gamma, gamma, exponential, chi-square, the Weibull, the Maxwell-Boltzmann, the Rayleigh, and related densities. $c_{1}, c_{2}$, and $c_{3}$ defined in (10), (11), and (12), respectively, are the normalizing constants if we consider statistical densities.

By a suitable modification of the Maxwell-Boltzmann distribution given in (3) through the pathway model, we get a more general energy distribution called the pathway energy distribution given by the density

$$
\begin{aligned}
f_{\mathrm{PD}}(E) \mathrm{d} E= & \frac{2 \pi(q-1)^{3 / 2}}{(\pi k T)^{3 / 2}} \frac{\Gamma(1 /(q-1))}{\Gamma(1 /(q-1)-3 / 2)} \\
& \times \sqrt{E}\left[1+(q-1) \frac{E}{k T}\right]^{-1 /(q-1)} \mathrm{d} E,
\end{aligned}
$$

for $q>1,1 /(q-1)-3 / 2>0$. The Maxwell-Boltzmann energy distribution can be retrieved from (13) by taking $q \rightarrow 1$. Thus the reaction rate probability integral given in (4) can be modified by using (13) and we get the extended reaction rate as

$$
\begin{aligned}
\widetilde{r}_{12}= & \left(1-\frac{1}{2} \delta_{12}\right) n_{1} n_{2}\left(\frac{8}{\pi \mu}\right)^{1 / 2}\left(\frac{q-1}{k T}\right)^{3 / 2} \\
& \times \frac{\Gamma(1 /(q-1))}{\Gamma(1 /(q-1)-3 / 2)} \sum_{\nu=0}^{2} \frac{S^{(\nu)}(0)}{\nu !} \\
& \times \int_{0}^{\infty} E^{\nu}\left[1+(q-1) \frac{E}{k T}\right]^{-1 /(q-1)} \\
& \times \exp \left[-\left(\frac{E_{G}}{E}\right)^{1 / 2}\right] \mathrm{d} E
\end{aligned}
$$

for $q>1,1 /(q-1)-3 / 2>0$. Substituting $y=E / k T$ and $x=$ $\left(E_{G} / k T\right)^{1 / 2}$ we obtain the above integral in a more convenient form as follows:

$$
\begin{aligned}
\widetilde{r}_{12}= & \left(1-\frac{1}{2} \delta_{12}\right) n_{1} n_{2}\left(\frac{8}{\pi \mu}\right)^{1 / 2}(q-1)^{3 / 2} \\
& \times \frac{\Gamma(1 /(q-1))}{\Gamma(1 /(q-1)-3 / 2)} \sum_{\nu=0}^{2}\left(\frac{1}{k T}\right)^{-\nu+(1 / 2)} \frac{S^{(\nu)}(0)}{\nu !} \\
& \times \int_{0}^{\infty} y^{\nu}[1+(q-1) y]^{-1 /(q-1)} \mathrm{e}^{-x y^{-1 / 2}} \mathrm{~d} y,
\end{aligned}
$$

for $q>1,1 /(q-1)-3 / 2>0$. Here we consider the integral to be evaluated as

$$
\begin{aligned}
I_{1 q}=\int_{0}^{\infty} y^{\nu}[1+(q-1) y]^{-1 /(q-1)} \\
\\
\times \mathrm{e}^{-x y^{-1 / 2}} \mathrm{~d} y .
\end{aligned}
$$

The integral can be evaluated by the techniques in applied analysis and can be obtained in closed form via Meijer's $G$ function as $[2,23,24]$

$$
\begin{aligned}
I_{1 q}= & \frac{(\pi)^{-1 / 2}}{(q-1)^{\nu+1} \Gamma(1 /(q-1))} \\
& \times G_{1,3}^{3,1}\left(\left.\frac{(q-1) x^{2}}{4}\right|_{0,1 / 2, v+1} ^{2-1 /(q-1)+v}\right)
\end{aligned}
$$

which yields the nonresonant reaction rate probability integral in the extended case as

$$
\begin{aligned}
& \tilde{r}_{12}=\left(1-\frac{1}{2} \delta_{12}\right) n_{1} n_{2}\left(\frac{8}{\mu}\right)^{1 / 2} \frac{\pi^{-1}}{\Gamma(1 /(q-1)-3 / 2)} \\
& \times \sum_{\nu=0}^{2}\left(\frac{q-1}{k T}\right)^{-v+(1 / 2)} \frac{S^{(\nu)}(0)}{\nu !} \\
& \times G_{1,3}^{3,1}\left[\left.\frac{(q-1) E_{G}}{4 k T}\right|_{0,1 / 2, v+1} ^{2-1 /(q-1)+v}\right] .
\end{aligned}
$$


Meijer's $G$-function and its properties can be seen in Mathai and Saxena [25] and Mathai [26]. We can obtain series expansions of the $G$-function given in (18) by combining the theories of residue calculus and generalized special functions; see Kumar and Haubold [24] for series expansions for all possible values of $\nu$. In many cases the nuclear factor $S^{(\nu)}(0)$ is approximately constant across the fusion window. Taking $S^{(v)}(0)=0$ for $v=1$ and $\nu=2$ and taking $S^{0}(0)=S(0)$, we obtain the extended reaction rate probability integral as

$$
\begin{aligned}
\tilde{r}_{12}= & \left(1-\frac{1}{2} \delta_{12}\right) n_{1} n_{2}\left[\frac{8(q-1)}{\mu k T}\right]^{1 / 2} \frac{\pi^{-1}}{\Gamma(1 /(q-1)-3 / 2)} \\
& \times S(0) G_{1,3}^{3,1}\left[\left.\frac{(q-1) E_{G}}{4 k T}\right|_{0,1 / 2,1} ^{2-1 /(q-1)}\right] .
\end{aligned}
$$

The series representation for (19) can be obtained as

$$
\begin{aligned}
\tilde{r}_{12}=\left(1-\frac{1}{2} \delta_{12}\right) n_{1} n_{2}\left[\frac{8(q-1)}{\mu k T}\right]^{1 / 2} \frac{\pi^{-1}}{\Gamma(1 /(q-1)-3 / 2)} \\
\times S(0)\{\sqrt{\pi} \Gamma(1 /(q-1)-1) \\
-2 \pi \Gamma\left(\frac{1}{q-1}-\frac{1}{2}\right)\left[\frac{(q-1) E_{G}}{4 k T}\right]^{1 / 2} \\
\times{ }_{1} F_{2}\left(\frac{1}{q-1}-\frac{1}{2} ; \frac{3}{2}, \frac{1}{2} ;-\frac{(q-1) E_{G}}{4 k T}\right) \\
+\left(\frac{2 \sqrt{\pi}(q-1) E_{G}}{4 k T}\right) \\
\times \sum_{r=0}^{\infty}\left(\frac{(q-1) E_{G}}{4 k T}\right)^{r} \\
\left.\times\left[A_{r}-\ln \left(\frac{(q-1) E_{G}}{4 k T}\right)\right] B_{r}\right\},
\end{aligned}
$$

where

$$
\begin{aligned}
A_{r}= & \Psi\left(-\frac{1}{2}-r\right)+\Psi\left(\frac{1}{q-1}+r\right) \\
& +\Psi(1+r)+\Psi(2+r), \\
B_{r}= & \frac{(-1)^{r} \Gamma(1 /(q-1)+r)}{(3 / 2)_{r} r !(1+r) !} .
\end{aligned}
$$

See the Appendix for detailed evaluation. As $q \rightarrow 1$ in (19), then, by using Stirling's formula for gamma functions given by

$$
\begin{gathered}
\Gamma(z+a) \approx(2 \pi)^{1 / 2} z^{z+a-1 / 2} \mathrm{e}^{-z} \\
|z| \longrightarrow \infty, \quad a \text { is bounded }
\end{gathered}
$$

we get the reaction rate probability integral in the MaxwellBoltzmann case as

$$
\begin{aligned}
r_{12}= & \left(1-\frac{1}{2} \delta_{12}\right) n_{1} n_{2}\left(\frac{8}{\pi \mu}\right)^{1 / 2}\left(\frac{1}{k T}\right)^{3 / 2} S(0) \\
& \times \int_{0}^{\infty} \exp \left[-\frac{E}{k T}-\left(\frac{E_{G}}{E}\right)^{1 / 2}\right] \mathrm{d} E \\
= & \left(1-\frac{1}{2} \delta_{12}\right) n_{1} n_{2}\left(\frac{8}{\mu k T}\right)^{1 / 2} S(0) \pi^{-1} \\
& \times G_{0,3}^{3,0}\left[\left.\frac{E_{G}}{4 k T}\right|_{0,1 / 2,1} ^{-}\right]
\end{aligned}
$$

which is obtained in a series of papers by Mathai and Haubold; see, for example, Mathai and Haubold [5]. The integral in (23) is dominated by the minimum value of $E / k T+$ $\left(E_{G} / E\right)^{1 / 2}=g(E)$ (say). The minimum value of the function $g(E)$, say $E_{0}$, can be determined as

$$
\begin{aligned}
& \frac{\mathrm{d}}{\mathrm{d} E}\left[\frac{E}{k T}+\left(\frac{E_{G}}{E}\right)^{1 / 2}\right]_{E=E_{0}} \\
& \quad=\frac{1}{k T}-\frac{1}{2} E_{G}^{1 / 2} E_{0}^{-3 / 2}=0 \Longrightarrow E_{0}=E_{G}^{1 / 2}\left(\frac{k T}{2}\right)^{3 / 2}
\end{aligned}
$$

and the function

$$
g\left(E_{0}\right)=3\left(\frac{E_{G}}{4 k T}\right)^{1 / 3}=3 \Theta,
$$

where $\Theta=\left(E_{G} / 4 k T\right)^{1 / 3}$. Now by using the Laplace method $[27,28]$ we can obtain an approximate value for $(23)$ as

$$
r_{12} \approx\left(1-\frac{1}{2} \delta_{12}\right) n_{1} n_{2} \frac{8 S(0) \Theta^{2} \exp (-3 \Theta)}{\sqrt{3} \pi \mu \alpha Z_{1} Z_{2} c} .
$$

In the next section we will obtain the mass, pressure, and temperature for the case of analytic stellar models characterized by density distribution and corresponding temperature distribution suggested by Haubold and Mathai [11].

\section{Closed Forms of the Integral over the Stellar Nuclear Energy Generation Rate}

Let us consider the density distribution $\varrho(r)$ considered by Haubold and Mathai $[6,11]$ and Mathai and Haubold [5] in the form

$$
\varrho(r)=\varrho_{c}\left[1-\left(\frac{r}{R}\right)^{\delta}\right], \quad \delta>0,
$$

where $\varrho_{c}$ is the central density of the star, $r$ is an arbitrary distance from the center, and $R$ is the solar radius. This density function is capable of producing different density distributions by choosing the free parameter $\delta$. Now we determine the quantities $M(r), P(r)$, and $T(r)$, the mass, the pressure, and the temperature at $r$. 
By the equation of the mass conservation

$$
\frac{\mathrm{d} M(r)}{\mathrm{d} r}=4 \pi r^{2} \varrho(r)
$$

we get

$$
\begin{aligned}
M(r) & =4 \pi \varrho_{c} \int_{0}^{r} t^{2}\left[1-\left(\frac{t}{R}\right)^{\delta}\right] \mathrm{d} t \\
& =\frac{4 \pi}{3} \varrho_{c} r^{3}\left[1-\frac{3}{\delta+3}\left(\frac{r}{R}\right)^{\delta}\right] .
\end{aligned}
$$

From (30), we get the central density as

$$
\varrho_{c}=\frac{3(\delta+3)}{4 \pi \delta} \frac{M(R)}{R^{3}} .
$$

If an element of a matter at a distance $r$ from the center of a spherical system is in hydrostatic equilibrium, then setting the sum of the radial forces acting on it to zero we obtain

$$
\frac{\mathrm{d} P(r)}{\mathrm{d} r}=-\frac{G \varrho(r) M(r)}{r^{2}},
$$

where $G$ is the gravitational constant. Assuming that the pressure at the center of the sun is $P_{c}$ and at the surface is zero, we get

$$
\begin{gathered}
P(r)=P_{c}-G \int_{0}^{r} \frac{M(t) \varrho(t)}{t^{2}} \mathrm{~d} t \\
=\frac{4 \pi}{3} G \varrho_{c}^{2} R^{2}\left[\xi-\frac{1}{2}\left(\frac{r}{R}\right)^{2}+\frac{\delta+6}{(\delta+2)(\delta+3)}\left(\frac{r}{R}\right)^{\delta+2}\right. \\
\left.\quad-\frac{3}{2(\delta+1)(\delta+3)}\left(\frac{r}{R}\right)^{2 \delta+2}\right] .
\end{gathered}
$$

Using the boundary conditions $P(R)=0$, we get $P_{c}=$ $(4 \pi / 3) G \xi \varrho_{c}^{2} R^{2}$, where

$$
\xi=\frac{1}{2}-\frac{\delta+6}{(\delta+2)(\delta+3)}+\frac{3}{2(\delta+1)(\delta+3)} .
$$

By the kinetic theory of gases, for a perfect gas, the pressure is given by

$$
P(r)=\frac{k N_{A}}{\mu} \varrho(r) T(r) .
$$

For the temperature of interest for stellar models, we neglect the negligible radiation pressure from the total pressure and obtain from (35) the following:

$$
\begin{aligned}
T(r)= & \frac{\mu}{k N_{A}} \frac{P(r)}{\varrho(r)} \\
= & \frac{4 \pi}{3 k N_{A}} \frac{G \mu \varrho_{c} R^{2}}{\left[1-(r / R)^{\delta}\right]} \\
& \times\left[\xi-\frac{1}{2}\left(\frac{r}{R}\right)^{2}+\frac{\delta+6}{(\delta+2)(\delta+3)}\left(\frac{r}{R}\right)^{\delta+2}\right. \\
& \left.\quad-\frac{3}{2(\delta+1)(\delta+3)}\left(\frac{r}{R}\right)^{2 \delta+2}\right] .
\end{aligned}
$$

The central temperature $T_{c}=\left(4 / k N_{A}\right) G \mu \xi(M(R) / R)$, where $\xi$ is as defined in (34).

Thus we have obtained the mass, the pressure, and the temperature throughout the nonlinear stellar model with the density distribution defined in (28). Next our aim is to obtain analytical results for stellar luminosity and neutrino emission rates for various stellar models.

\section{Stellar Luminosity and Neutrino Emission Rate}

The energy conservation equation states that the net increase in the rate of energy flux coming out of a spherical shell from the inside is the same as the energy produced within the shell [29]. If we denote $L_{r}=L(r)$ as the energy flux through the sphere of radius $r$, then we have

$$
\frac{\mathrm{d} L_{r}}{\mathrm{~d} r}=4 \pi r^{2} \varrho(r) \varepsilon(r),
$$

where $\varepsilon(r)$ is the energy produced per second by nuclear reactions in each gram of stellar matter. $\varepsilon(r)$ depends on the chemical composition in each gram of stellar matter. Here usually $L_{r}$ is a constant but will be equal to $L$ at the surface of the star. We assume here that the star is chemically homogeneous (that is a star where chemical composition throughout is a constant). Also we assume the energy generation rate $\varepsilon(r)$ for one particular nuclear reaction. Now if we denote $\widetilde{r}_{12}(\varrho(r), T(r))$ as the extended nonresonant thermonuclear reaction rate for the particles 1 and 2 defined by (19), then we will consider the energy generation rate $\varepsilon_{12}(r)$ and it can be written in terms of the extended reaction rates via

$$
\varepsilon_{12}(r)=\frac{1}{\varrho(r)} \mathscr{C}^{*} \varrho^{2} G_{1,3}^{3,1}\left[\left.\frac{(q-1) E_{G}}{4 k T}\right|_{0,1 / 2,1} ^{2-1 /(q-1)}\right],
$$

where

$$
\mathscr{C}^{*}=\frac{E_{12} \widetilde{r}_{12}(\varrho(r), T(r))}{\varrho^{2} G_{1,3}^{3,1}\left[(q-1) E_{G} /\left.4 k T\right|_{0,1 / 2,1} ^{2-1 /(q-1)}\right]}
$$

in which $E_{12}$ is the amount of energy given off in a single reaction. It is to be noted that, by using the asymptotic behaviour of $G_{1,3}^{3,1}\left((q-1) E_{G} / 4 k T\right)$ [26] and as $q \rightarrow 1$, $\mathscr{C}^{*} \rightarrow \mathscr{C}$, the composite parameter considered by [9], which is defined as

$$
\mathscr{C}=\frac{E_{12} r_{12}}{\varrho^{2} \Theta^{2}} \exp (3 \Theta)
$$

for our universe $\mathscr{C} \approx 2 \times 10^{4}$ for proton-proton fusion under typical stellar conditions [9]. Then from (37) we have the total luminosity of the star by integration as follows:

$$
L(R)=\int_{0}^{R} 4 \pi r^{2} \varrho(r) \varepsilon(r) \mathrm{d} r .
$$

If we are considering only one specific reaction defined as in (1), then we have

$$
L_{12}(R)=\int_{0}^{R} 4 \pi r^{2} \varrho(r) \varepsilon_{12}(r) \mathrm{d} r,
$$


where the energy generation rate is defined in (38) and $\varrho(r)$ is a suitable density distribution explaining the sun. Writing (42) in terms of $\widetilde{r}_{12}(\varrho(r), T(r))$ we get

$$
\begin{aligned}
L_{12}(R) & =\int_{0}^{R} 4 \pi r^{2} \mathscr{C}^{*} \varrho^{2} G_{1,3}^{3,1}\left[\left.\frac{(q-1) E_{G}}{4 k T}\right|_{0,1 / 2,1} ^{2-1 /(q-1)}\right] \mathrm{d} r \\
& =\int_{0}^{R} 4 \pi r^{2} E_{12} \widetilde{r}_{12}(\varrho(r), T(r)) \mathrm{d} r .
\end{aligned}
$$

The number density $n_{i}$ of a particle $i$, for a gas of mean density $\varrho(r)$, can be expressed as

$$
n_{i}(r)=\varrho(r) N_{A} \frac{X_{i}}{A_{i}},
$$

where $N_{A}$ stands for Avagadro's constant, $A_{i}$ is the atomic mass of particle $i$ in atomic mass units, and $X_{i}$ is the mass fraction of particle $i$ such that $\sum_{i} X_{i}=1$. Substituting $\tilde{r}_{12}(\varrho(r), T(r))$ from (19) and using (44) we have

$$
\begin{aligned}
& L_{12}(R) \\
& =\int_{0}^{R} 4 \pi r^{2} E_{12}\left(1-\frac{1}{2} \delta_{12}\right) N_{A}^{2} \varrho^{2}(r) \frac{X_{1} X_{2}}{A_{1} A_{2}}\left[\frac{8(q-1)}{\mu k T(r)}\right]^{1 / 2} \\
& \quad \times \frac{\pi^{-1}}{\Gamma(1 /(q-1)-3 / 2)} S(0) G_{1,3}^{3,1} \\
& \quad \times\left[\left.\frac{(q-1) \pi^{2} \mu}{2 k T(r)}\left(\frac{Z_{1} Z_{2} e^{2}}{\hbar}\right)^{2}\right|_{0,1 / 2,1} ^{2-1 /(q-1)}\right] \mathrm{d} r .
\end{aligned}
$$

If we divide the "internal luminosity" $L_{12}\left(R_{\odot}\right)$ by the amount of energy $E_{12}$, then we get the total number of particles per second $N_{12}$ liberated in the reaction given by (1) as follows:

$$
\begin{aligned}
N_{12}= & \frac{L_{12}(R)}{E_{12}} \\
= & 4\left(1-\frac{1}{2} \delta_{12}\right) N_{A}^{2} \frac{X_{1} X_{2}}{A_{1} A_{2}}\left[\frac{8(q-1)}{\mu k}\right]^{1 / 2} \\
& \times \frac{1}{\Gamma(1 /(q-1)-3 / 2)} S(0) \\
& \times \int_{0}^{R} \frac{r^{2} \varrho^{2}(r)}{[T(r)]^{1 / 2}} G_{1,3}^{3,1}\left[\frac{(q-1) \pi^{2} \mu}{2 k T(r)}\right. \\
& \left.\quad \times\left.\left(\frac{Z_{1} Z_{2} e^{2}}{\hbar}\right)^{2}\right|_{0,1 / 2,1} ^{2-1 /(q-1)}\right] \mathrm{d} r
\end{aligned}
$$

$$
\begin{aligned}
& =4\left(1-\frac{1}{2} \delta_{12}\right) N_{A}^{2} \frac{X_{1} X_{2}}{A_{1} A_{2}}\left[\frac{8(q-1)}{\mu k}\right]^{1 / 2} \\
& \times \frac{1}{\Gamma(1 /(q-1)-3 / 2)} S(0) \frac{1}{2 \pi i} \\
& \times \int_{L} \Gamma(s) \Gamma\left(\frac{1}{2}+s\right) \Gamma(1+s) \\
& \quad \times \Gamma\left(\frac{1}{q-1}-1-s\right)\left[\frac{(q-1) \pi^{2} \mu}{2 k}\left(\frac{Z_{1} Z_{2} \mathrm{e}^{2}}{\hbar}\right)^{2}\right]^{-s} \\
& \quad \times \int_{0}^{R} r^{2} \varrho^{2}(r)[T(r)]^{-1 / 2+s} \mathrm{~d} r \mathrm{~d} s .
\end{aligned}
$$

For the density distribution defined in (28) introduced by Haubold and Mathai $[5,6]$ and the corresponding temperature distribution (36), we get

$$
\begin{aligned}
& \int_{0}^{R} r^{2} \varrho^{2}(r)[T(r)]^{-1 / 2+s} \mathrm{~d} r \\
& =\left[\frac{4 \pi G \mu}{3 k N_{A}} \varrho_{c} R^{2}\right]^{s-1 / 2} \varrho_{c}^{2} \\
& \times \int_{0}^{R} r^{2}\left[1-\left(\frac{r}{R}\right)^{\delta}\right]^{5 / 2-s} \\
& \times\left[-\frac{1}{2}\left(\frac{r}{R}\right)^{2}+\frac{\delta+6}{(\delta+2)(\delta+3)}\left(\frac{r}{R}\right)^{\delta+2}\right. \\
& \left.\quad-\frac{3}{2(\delta+1)(\delta+3)}\left(\frac{r}{R}\right)^{2 \delta+2}\right]^{s-1 / 2} \mathrm{~d} r
\end{aligned}
$$

where $\xi$ is as defined in (34). If we put a substitution $r=x R$, then we get

$$
\begin{aligned}
& \int_{0}^{R} r^{2} \varrho^{2}(r)[T(r)]^{-1 / 2+s} \mathrm{~d} r \\
& =\left[\frac{4 \pi G \mu}{3 k N_{A}} \varrho_{c} R^{2}\right]^{s-1 / 2} \varrho_{c}^{2} R^{3} \\
& \times \int_{0}^{1} x^{2}\left[1-x^{\delta}\right]^{5 / 2-s} \\
& \times\left[\xi-\frac{1}{2} x^{2}+\frac{\delta+6}{(\delta+2)(\delta+3)} x^{\delta+2}\right. \\
& \left.\quad-\frac{3}{2(\delta+1)(\delta+3)} x^{2 \delta+2}\right]^{s-1 / 2} \mathrm{~d} x .
\end{aligned}
$$


Putting $x^{\delta}=y$ and simplifying we obtain

$$
\begin{aligned}
& \int_{0}^{R} r^{2} \varrho^{2}(r)[T(r)]^{-1 / 2+s} \mathrm{~d} r \\
& =\left[\frac{4 \pi G \mu \xi}{3 k N_{A}} \varrho_{c} R^{2}\right]^{s-1 / 2} \frac{\varrho_{c}^{2} R^{3}}{\delta} \\
& \quad \times \int_{0}^{1} y^{3 / \delta-1}[1-y]^{5 / 2-s}[1-u(y)]^{s-1 / 2} \mathrm{~d} y,
\end{aligned}
$$

where $u(y)$ is defined as

$$
u(y)=\frac{y^{2 / \delta}}{\xi}\left[\frac{1}{2}-\frac{\delta+6}{(\delta+2)(\delta+3)} y+\frac{3}{2(\delta+1)(\delta+3)} y^{2}\right]
$$

As $y \rightarrow 0, u(y) \rightarrow 0$, and $y \rightarrow 1, u(y) \rightarrow 1$. If we take

$$
v(y)=\frac{1}{2}-\frac{\delta+6}{(\delta+2)(\delta+3)} y+\frac{3}{2(\delta+1)(\delta+3)} y^{2},
$$

we have $v(0)=1 / 2$. The minimum value of $v(y)$ is at $y=$ $(\delta+1)(\delta+6) / 3(\delta+2)$ and the value is $1 / 2-1 / 6\left((\delta+6)^{2}(\delta+\right.$ $\left.1) /(\delta+3)(\delta+2)^{2}\right)$. Thus the minimum value is nonnegative since $(\delta+6)^{2}(\delta+1) /(\delta+3)(\delta+2)^{2}$ decreases steadily from 3 to 1 for all $\delta>0$. Therefore $v(y) \leq 0$. Since $\xi>0$, for all $\delta>0, u(y) \leq 0$ for all $\delta>0, \xi>0$. Thus $[1-u(y)]^{s-1 / 2} \leq 0$. Hence $0<u(y)<1$ for $0<y<1$ and for $\delta>0$. Thus by using the binomial expansion we obtain

$$
[1-u(y)]^{s-1 / 2}=\sum_{m=0}^{\infty} \frac{(1 / 2-s)_{m}}{m !}[u(y)]^{m}
$$

where $(1 / 2-s)_{m}$ is the Pochhammer symbol defined for $a \in$ $\mathbb{C}$ by

$$
\begin{aligned}
(a)_{0} & =1, \\
(a)_{m} & =\left\{\begin{array}{l}
a(a+1) \cdots(a+m-1), \\
\frac{\Gamma(a+m)}{\Gamma(a)},
\end{array}\right.
\end{aligned}
$$

whenever $\Gamma(a)$ exists. Taking $\delta=2$ we get $\xi=1 / 5$ and

$$
[1-u(y)]^{s-1 / 2}=(1-y)^{2 s-1}\left(1-\frac{1}{2} y\right)^{s-1 / 2}
$$

Then from (49) we obtain

$$
\begin{aligned}
& \int_{0}^{R} r^{2} \varrho^{2}(r)[T(r)]^{-1 / 2+s} \mathrm{~d} r \\
& =\left[\frac{4 \pi G \mu}{15 k N_{A}} \varrho_{c} R^{2}\right]^{s-1 / 2} \frac{\varrho_{c}^{2} R^{3}}{2} \\
& \quad \times \int_{0}^{1} y^{3 / 2-1}[1-y]^{5 / 2+s-1}\left(1-\frac{1}{2} y\right)^{s-1 / 2} \mathrm{~d} y \\
& =\left[\frac{4 \pi G \mu}{15 k N_{A}} \varrho_{c} R^{2}\right]^{s-1 / 2} \frac{\varrho_{c}^{2} R^{3}}{2} \\
& \quad \times \sum_{m=0}^{\infty} \frac{(1 / 2-s)_{m}}{m !} \frac{1}{2^{m}} \\
& \quad \times \int_{0}^{1} y^{3 / 2+m-1}[1-y]^{5 / 2+s-1} \mathrm{~d} y .
\end{aligned}
$$

By using beta integral and using (53) we obtain

$$
\begin{aligned}
& \int_{0}^{R} r^{2} \varrho^{2}(r)[T(r)]^{-1 / 2+s} \mathrm{~d} r \\
& =\left[\frac{4 \pi G \mu}{15 k N_{A}} \varrho_{c} R^{2}\right]^{s-1 / 2} \frac{\varrho_{c}^{2} R^{3}}{2} \\
& \quad \times \sum_{m=0}^{\infty} \frac{\Gamma(1 / 2-s+m)}{m ! \Gamma(1 / 2-s)} \frac{1}{2^{m}} \frac{\Gamma(3 / 2+m) \Gamma(5 / 2+s)}{\Gamma(4+s+m)} .
\end{aligned}
$$

Now from (46) we obtain the total number of particles per second liberated in the reaction (1) as follows:

$$
\begin{aligned}
N_{12}= & \frac{2 N_{A}^{2} \varrho_{c}^{2} R^{2}}{\mu}\left(1-\frac{1}{2} \delta_{12}\right) \frac{X_{1} X_{2}}{A_{1} A_{2}}\left[\frac{30(q-1) N_{A}}{\pi G \varrho_{c}}\right]^{1 / 2} \\
& \times S(0) \frac{1}{\Gamma(1 /(q-1)-3 / 2)} \\
& \times \sum_{m=0}^{\infty} \frac{1}{2^{m}} \frac{\Gamma(3 / 2+m)}{m !} \frac{1}{2 \pi i} \\
& \times \int_{L} \Gamma(s) \Gamma\left(\frac{1}{2}+s\right) \Gamma(1+s) \Gamma\left(\frac{5}{2}+s\right) \\
& \times \frac{\Gamma(1 /(q-1)-1-s) \Gamma(1 / 2+m-s)}{\Gamma(1 / 2-s) \Gamma(4+m+s)} \\
& \times\left[\frac{15 \pi(q-1) N_{A}}{8 G \varrho_{c} R^{2}}\left(\frac{Z_{1} Z_{2} \mathrm{e}^{2}}{\hbar}\right)^{2}\right]^{-s} \mathrm{~d} s
\end{aligned}
$$




$$
\begin{aligned}
N_{12}= & \frac{2 N_{A}^{2} \varrho_{c}^{2} R^{2}}{\mu}\left(1-\frac{1}{2} \delta_{12}\right) \frac{X_{1} X_{2}}{A_{1} A_{2}}\left[\frac{30(q-1) N_{A}}{\pi G \varrho_{c}}\right]^{1 / 2} \\
& \times S(0) \frac{1}{\Gamma(1 /(q-1)-3 / 2)} \\
& \times \sum_{m=0}^{\infty} \frac{1}{2^{m}} \frac{\Gamma(3 / 2+m)}{m !} \\
& \times G_{3,5}^{4,2}\left[\frac{15 \pi(q-1) N_{A}}{8 G \varrho_{c} R^{2}}\right. \\
& \left.\times\left.\left(\frac{Z_{1} Z_{2} \mathrm{e}^{2}}{\hbar}\right)^{2}\right|_{0,1 / 2,1,5 / 2,1 / 2} ^{2-1 /(q-1), 1 / 2-m, 4+m}\right] .
\end{aligned}
$$

For more details on $G$-function and its properties see [25, 26]. Thus we have obtained the the total number of particles per second liberated in the reaction (1) in terms of the density distribution considered by Haubold and Mathai [6, 29].

\section{Comparison of Pathway Energy Density and the Maxwell-Boltzmann Energy Density}

In Figure 1 it can be obtserved that, for the nuclei to react at energy $E$, they have to borrow an energy $E$ from the thermal environment. The probability of such an energy is proportional to the Maxwell-Boltzmann energy $\exp [-E / k T]$. The fusion will take place when the nuclei penerate the Coulomb barrier keeping them apart. The probability of penetration is given by the factor $\exp \left[-\left(E_{G} / E\right)^{1 / 2}\right]$. The product of these two factors illustrates that fusion mostly occurs in the energy window given in the figure.

In Figure 2 the pathway energy density is plotted for $q=$ $0.7,0.9,1,1.2,1.4$, respectively. For different values of $q$ we get different energy densities (curves (a), (b), (c), (d), and (e)). The nonresonant cross section is also plotted. The product of the pathway energy density and the nonresonant cross section for different values of $q$, namely, $q=0.7,0.9,1,1.2,1.4$, is also plotted. It is to be noted that as $q \rightarrow 1$ the pathway energy density coincides with the Maxwell-Boltzmann energy density and also the fusion window for the Maxwell-Boltzmann case in Figure 1.

The curves in the figure represent pathway density $[1+(q-1)(E / k T)]^{-1 /(q-1)}$ for (a) $q=0.7$, (b) $q=0.9$, (c) $q=1$, (d) $q=1.2$, and (e) $q=1.4$. (f) represents the nonresonant cross section $\exp \left[-\left(E_{G} / E\right)^{1 / 2}\right]$. The product $[1+(q-1)(E / k T)]^{-1 /(q-1)} \exp \left[-\left(E_{G} / E\right)^{1 / 2}\right]$ is also represented for (g) $q=0.7$, (h) $q=0.9$, (i) $q=1$, (j) $q=1.2$, and $(\mathrm{k}) q=1.4$.

Figure 3(a) shows the pathway energy density defined in (13) for $q=1,1.2,1.3,1.4$ and Figure 3(b) shows the MaxwellBoltzmann energy density defined in (3). As $q \rightarrow 1$ the pathway energy density reduces to the Maxwell-Boltzmann energy density. The pathway energy density covers many stable and unstable situations as the value of $q$ varies. If

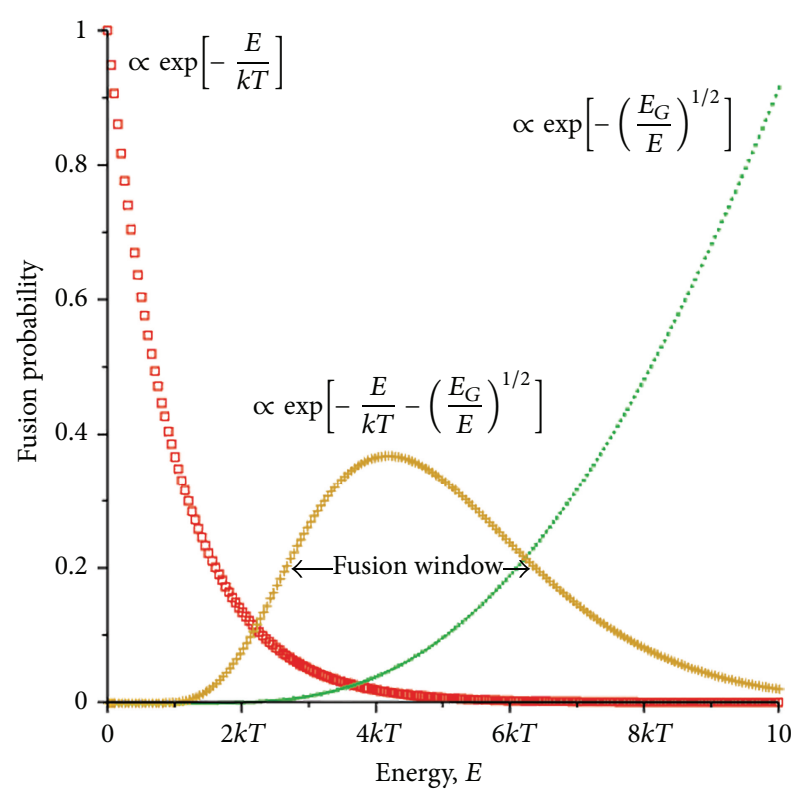

FIgURE 1: Schematic plot of the enrgy-dependent factors for the reaction rate probability integral: the Maxwell-Boltzmann energy density, nonresonant nuclear cross section, and the product of the Maxwell-Boltzmann density and the nonresonant cross section.

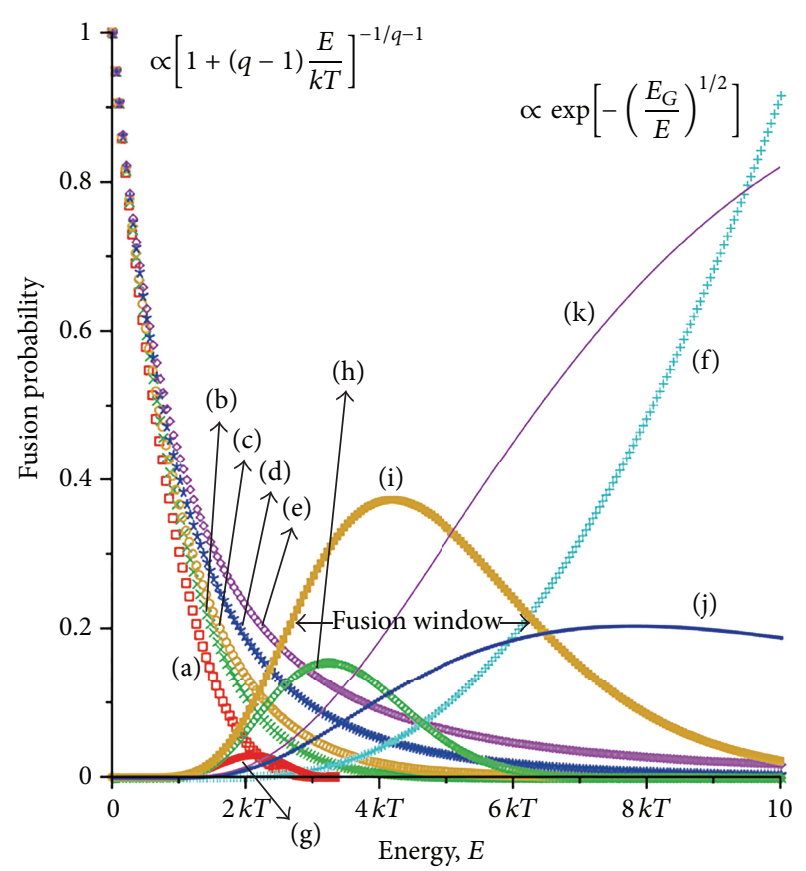

FIGURE 2: Schematic plot of the energy-dependent factors for the extended reaction rate probability integral: pathway energy density, nonresonant nuclear cross section, and the product of the pathway density and the nonresonant cross section.

the Maxwell-Boltzmann density is the equilibrium situation, many other nonequilibrium situations are covered by the pathway energy density. 


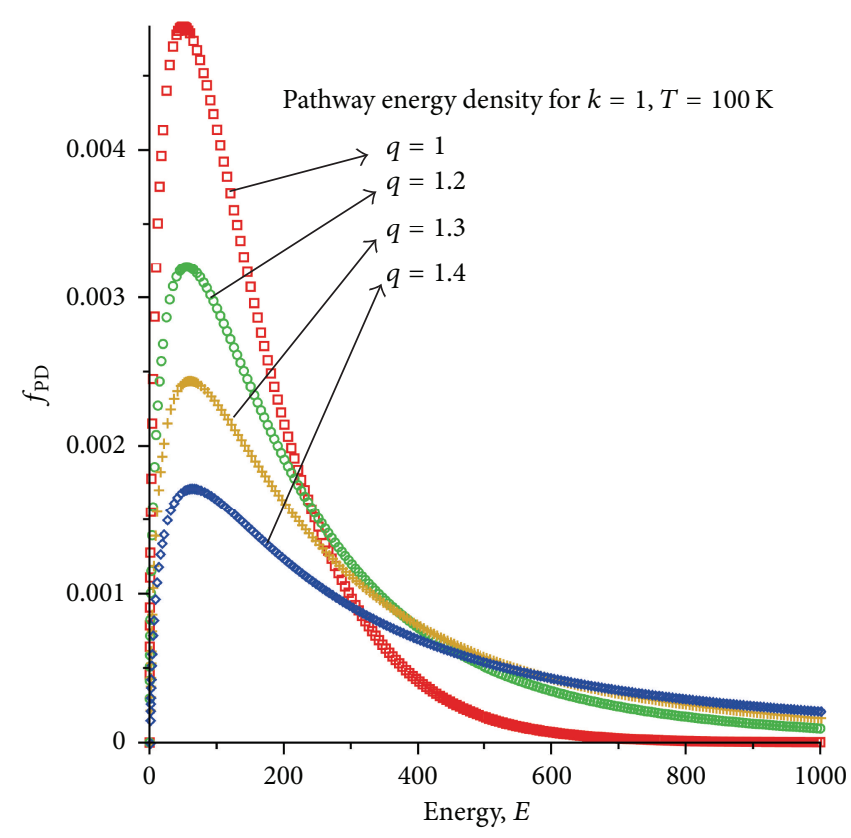

(a)

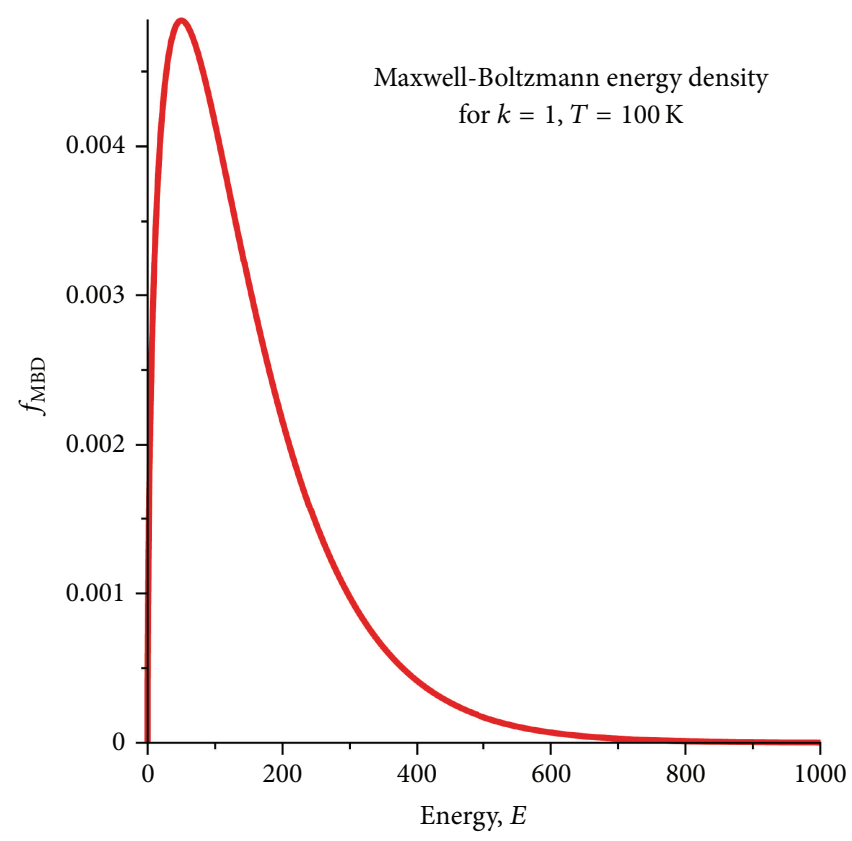

(b)

Figure 3: (a) Pathway energy density for $q=1,1.2,1.3,1.4$ and for $k=1, T=100 \mathrm{~K}$. (b) The Maxwell-Boltzmann energy density for $k=1, T=100 \mathrm{~K}$.

\section{Concluding Remarks}

In this paper we have modified the energy distribution for a nonresonant reaction rate probability integral. The composition parameter $\mathscr{C}$ considered by [9] is extended to $\mathscr{C}^{*}$ by the pathway energy density. Considering the analytic density distributions developed by Haubold and Mathai $[6$, 29], they are used to obtain the stellar luminosity and the neutrino emission rates and are obtained in generalized special functions such as Meijer's $G$-function. The pathway energy density considered here covers many density functions and hence the extended reaction rate integral covers a wider class of integral. Pathway energy density helps us to obtain various fusion windows by giving different values to $q$, the pathway parameter, which in turn leads to a new opening in the fusion research. The graphs plotted here are by using Maple 14 in Windows XP platform.

\section{Appendix}

\section{Series Representation}

The series representation for the right-hand side of (19) can be obtained through the following procedure. Here we apply residue calculus on the $G$-function given in (19). Consider the $G$-function as follows:

$$
G_{1,3}^{3,1}\left(\left.\frac{(q-1) E_{G}}{4 k T}\right|_{0,1 / 2,1} ^{2-1 /(q-1)}\right)
$$

$$
\begin{aligned}
& =\frac{1}{2 \pi i} \int_{c-i \infty}^{c+i \infty} \Gamma(s) \Gamma\left(\frac{1}{2}+s\right) \Gamma(1+s) \\
& \quad \times \Gamma\left(\frac{1}{q-1}-1-s\right)\left(\frac{(q-1) E_{G}}{4 k T}\right)^{-s} \mathrm{~d} s .
\end{aligned}
$$

The right-hand side is the sum of the residues of the integrand. The poles of the gammas in the integral representation in (19) are as follows:

$$
\begin{aligned}
& \text { poles of } \Gamma(s): s=0,-1,-2, \ldots ; \\
& \text { poles of } \Gamma(1 / 2+s): s=-1 / 2,-3 / 2,-5 / 2, \ldots \text {; } \\
& \text { poles of } \Gamma(1+s): s=-1,-2,-3, \ldots
\end{aligned}
$$

Here the poles of $\Gamma(s)$ and $\Gamma(1+s)$ will coincide with each other at all points except at $s=0$. Note that the pole $s=0$ is a pole of order $1, s=-1 / 2,-3 / 2,-5 / 2, \ldots$ are each of order 1 , and $s=-1,-2,-3, \ldots$ are each of order 2 . We know that

$$
\begin{aligned}
\lim _{s \rightarrow-r}(s+r) \Gamma(s) & =\frac{(-1)^{r}}{r !}, \\
\Gamma(a-r) & =\frac{(-1)^{r} \Gamma(a)}{(1-a)_{r}}, \\
\Gamma(a+m) & =\Gamma(a)(a)_{m}
\end{aligned}
$$


when $\Gamma(a)$ is defined, $r=0,1,2, \ldots ; \Gamma(1 / 2)=\pi^{1 / 2}$,

$$
(a)_{r}= \begin{cases}a(a+1) \cdots(a+r-1) & \text { if } r \geq 1, a \neq 0 \\ 1 & \text { if } r=0 .\end{cases}
$$

The sum of the residues corresponding to the poles $s=0$ is given by

$$
R_{1}=\sqrt{\pi} \Gamma\left(\frac{1}{q-1}-1\right) .
$$

The sum of the residues corresponding to the poles $s=$ $-1 / 2,-3 / 2,-5 / 2, \ldots$ is

$$
\begin{aligned}
R_{2}=\sum_{r=0}^{\infty} \frac{(-1)^{r}}{r !} \Gamma\left(-\frac{1}{2}-r\right) \Gamma\left(\frac{1}{2}-r\right) \\
\quad \times \Gamma\left(\frac{1}{q-1}-\frac{1}{2}+r\right)\left[\frac{(q-1) E_{G}}{4 k T}\right]^{-1 / 2+r} \\
=-2 \pi \Gamma\left(\frac{1}{q-1}-\frac{1}{2}\right)\left[\frac{(q-1) E_{G}}{4 k T}\right]^{1 / 2} \\
\quad \times{ }_{1} F_{2}\left(\frac{1}{q-1}-\frac{1}{2} ; \frac{3}{2}, \frac{1}{2} ;-\frac{(q-1) E_{G}}{4 k T}\right)
\end{aligned}
$$

where ${ }_{1} F_{2}$ is the hypergeometric function defined by

$$
{ }_{1} F_{2}(a ; b, c ; x)=\sum_{r=0}^{\infty} \frac{(a)_{r}}{(b)_{r}(c)_{r}} \frac{x^{r}}{r !} .
$$

To obtain the sum of the residues corresponding to poles $s=-1,-2,-3, \ldots$ of order 2 , we proceed as follows:

$$
\begin{aligned}
& \begin{aligned}
& R_{3=0} \sum_{s \rightarrow-1-r} \frac{\partial}{\partial s}[(s+1+r)^{2} \Gamma(1+s) \Gamma(s) \Gamma\left(\frac{1}{2}+s\right) \\
& \times\left.\Gamma\left(\frac{1}{q-1}-1-s\right)\left(\frac{(q-1) E_{G}}{4 k T}\right)^{-s}\right] \\
&=\sum_{r=0}^{\infty} \lim _{s \rightarrow-1-r} \frac{\partial}{\partial s}[\left(\Gamma^{2}(2+s+r) \Gamma(1 / 2+s)\right. \\
&\times \Gamma(1 /(q-1)-1-s)) \\
& \times\left((s+r)^{2}(s+r-1)^{2} \cdots(s+1)^{2} s\right)^{-1} \\
& \times\left.\left(\frac{(q-1) E_{G}}{4 k T}\right)^{-s}\right] \\
&=\sum_{r=0}^{\infty} \lim _{s \rightarrow-1-r} \frac{\partial}{\partial s} \Phi(s),
\end{aligned}
\end{aligned}
$$

where

$$
\begin{aligned}
\Phi(s)= & \frac{\Gamma^{2}(2+s+r) \Gamma(1 / 2+s) \Gamma(1 /(q-1)-1-s)}{(s+r)^{2}(s+r-1)^{2} \cdots(s+1)^{2} s} \\
& \times\left(\frac{(q-1) E_{G}}{4 k T}\right)^{-s} .
\end{aligned}
$$

We have

$$
\begin{aligned}
& \frac{\partial}{\partial s} \Phi(s)=\Phi(s) \frac{\partial}{\partial s}[\ln [\Phi(s)]] \\
& \ln \Phi(s)=2 \ln [\Gamma(2+s+r)]+\ln \left[\Gamma\left(\frac{1}{2}+s\right)\right] \\
&+\ln \left[\Gamma\left(\frac{1}{q-1}-1-s\right)\right]-s \ln \left(\frac{(q-1) E_{G}}{4 k T}\right) \\
&-2 \ln (s+r)-2 \ln (s+r-1)-\cdots \\
&-2 \ln (s+1)-\ln (s) \\
& \frac{\partial}{\partial s}[\ln [\Phi(s)]]= 2 \Psi(2+s+r)+\Psi\left(\frac{1}{2}+s\right) \\
&+\Psi\left(\frac{1}{q-1}-1-s\right) \\
&-\ln \left(\frac{(q-1) E_{G}}{4 k T}\right)-\frac{2}{s+r} \\
&-\frac{2}{s+r-1}-\cdots-\frac{2}{s+1}-\frac{1}{s} \\
& \lim _{s \rightarrow-1-r}\left\{\frac{\partial}{\partial s} \ln [\Phi(s)]\right\}= \Psi\left(-\frac{1}{2}-r\right)+\Psi\left(\frac{1}{q-1}+r\right) \\
&+\Psi(1+r)+\Psi(2+r) \\
&-\ln \left(\frac{(q-1) E_{G}}{4 k T}\right),
\end{aligned}
$$

where $\Psi(z)$ is a Psi function or digamma function (see Mathai [26]) and $\Psi(1)=-\gamma, \gamma=0.5772156649 \ldots$ is Euler's constant. Now

$$
\begin{aligned}
\lim _{s \rightarrow-1-r} \Phi(s)= & \frac{(-1)^{1+r} 2 \sqrt{\pi} \Gamma(1 /(q-1)+r)}{(3 / 2)_{r} r !(1+r) !} \\
& \times\left(\frac{(q-1) E_{G}}{4 k T}\right)^{1+r} .
\end{aligned}
$$


Then by using (A.7), (A.9), and (A.10) we get

$$
\begin{array}{r}
R_{3}=\sum_{r=0}^{\infty} \frac{(-1)^{1+r} 2 \sqrt{\pi} \Gamma(1 /(q-1)+r)}{(3 / 2)_{r} r !(1+r) !}\left(\frac{(q-1) E_{G}}{4 k T}\right)^{1+r} \\
\times\left[\Psi\left(-\frac{1}{2}-r\right)+\Psi\left(\frac{1}{q-1}+r\right)+\Psi(1+r)\right. \\
\left.+\Psi(2+r)-\ln \left(\frac{(q-1) E_{G}}{4 k T}\right)\right] \\
=\left(\frac{2 \sqrt{\pi}(q-1) E_{G}}{4 k T}\right) \\
\quad \times \sum_{r=0}^{\infty}\left(\frac{(q-1) E_{G}}{4 k T}\right)^{r}\left[A_{r}-\ln \left(\frac{(q-1) E_{G}}{4 k T}\right)\right] B_{r}
\end{array}
$$

where

$$
\begin{aligned}
A_{r}= & \Psi\left(-\frac{1}{2}-r\right)+\Psi\left(\frac{1}{q-1}+r\right) \\
& +\Psi(1+r)+\Psi(2+r), \\
B_{r}= & \frac{(-1)^{r} \Gamma(1 /(q-1)+r)}{(3 / 2)_{r} r !(1+r) !} .
\end{aligned}
$$

Thus from (A.4), (A.5), and (A.11) we get (20).

\section{Conflict of Interests}

The authors declare that there is no conflict of interests regarding the publication of this paper.

\section{Acknowledgments}

The authors would like to thank the Department of Science and Technology, Government of India, New Delhi, for the financial assistance for this work under Project no. SR/S4/MS:287/05 and the Centre for Mathematical Sciences for providing all facilities.

\section{References}

[1] R. Davis Jr., "Nobel Lecture: a half-century with solar neutrinos," Reviews of Modern Physics, vol. 75, no. 3, pp. 985-994, 2003.

[2] H. J. Haubold and D. Kumar, "Extension of thermonuclear functions through the pathway model including MaxwellBoltzmann and Tsallis distributions," Astroparticle Physics, vol. 29, no. 1, pp. 70-76, 2008.

[3] H. J. Haubold and R. W. John, "On the evaluation of an integral connected with the thermonuclear reaction rate in closedform," Astronomische Nachrichten, vol. 299, no. 5, pp. 225-232, 1978.

[4] W. A. Fowler, G. R. Caughlan, and B. A. Zimmerman, "Thermonuclear rection rates," Annual Review of Astronomy and Astrophysics, vol. 5, pp. 525-570, 1967.
[5] A. M. Mathai and H. J. Haubold, Modern Problems in Nuclear and Neutrino Astrophysics, Akademie, Berlin, Germany, 1988.

[6] H. J. Haubold and A. M. Mathai, "Analytic representations of modified non-resonant thermonuclear reaction rates," Journal of Applied Mathematics and Physics, vol. 37, no. 5, pp. 685-695, 1986.

[7] W. A. Fowler, "Experimental and theoretical nuclear astrophysics: the quest for the origin of the elements," Reviews of Modern Physics, vol. 56, no. 2, pp. 149-179, 1984.

[8] A. C. Phillips, The Physics of Stars, John Wiley \& Sons, Chichester, UK, 2nd edition, 1999.

[9] F. C. Adams, "Stars in other universes: stellar structure with different fundamental constants," Journal of Cosmology and Astroparticle Physics, vol. 2008, no. 8, article 10, 2008.

[10] M. Coraddu, G. Kaniadakis, A. Lavagno, M. Lissia, G. Mezzorani, and P. Quarati, "Thermal distributions in stellar plasmas, nuclear reactions and solar neutrinos," Brazilian Journal of Physics, vol. 29, no. 1, pp. 153-168, 1999.

[11] H. J. Haubold and A. M. Mathai, "On nuclear reaction rate theory," Annalen der Physik, vol. 41, pp. 380-396, 1984.

[12] M. Coraddu, M. Lissia, G. Mezzorani, and P. Quarati, "SuperKamiokande hep neutrino best fit: a possible signal of nonMaxwellian solar plasma," Physica A: Statistical Mechanics and Its Applications, vol. 326, no. 3-4, pp. 473-481, 2003.

[13] A. Lavagno and P. Quarati, "Classical and quantum nonextensive statistics effects in nuclear many-body problems," Chaos, Solitons and Fractals, vol. 13, no. 3, pp. 569-580, 2002.

[14] A. Lavagno and P. Quarati, "Metastability of electron-nuclear astrophysical plasmas: motivations, signals and conditions," Astrophysics and Space Science, vol. 305, no. 3, pp. 253-259, 2006.

[15] M. Lissia and P. Quarati, "Nuclear astrophysical plasmas: ion distribution functions and fusion rates," Europhysics News, vol. 36, no. 6, pp. 211-214, 2005.

[16] C. Tsallis, "Possible generalization of Boltzmann-Gibbs statistics," Journal of Statistical Physics, vol. 52, no. 1-2, pp. 479-487, 1988.

[17] C. Tsallis, Introduction to Non-Extensive Statistical Mechanics, Springer, New York, NY, USA, 2009.

[18] M. Gell-Mann and C. Tsallis, Eds., Nonextensive Entropy: Interdisciplinary Applications, Oxford University Press, New York, NY, USA, 2004.

[19] A. M. Mathai and H. J. Haubold, "Pathway model, superstatistics, Tsallis statistics, and a generalized measure of entropy," Physica A: Statistical Mechanics and Its Applications, vol. 375, no. 1, pp. 110-122, 2007.

[20] R. K. Saxena, A. M. Mathai, and H. J. Haubold, "Astrophysical thermonuclear functions for Boltzmann-Gibbs statistics and Tsallis statistics," Physica A: Statistical Mechanics and Its Applications, vol. 344, no. 3-4, pp. 649-656, 2004.

[21] A. M. Mathai, "A pathway to matrix-variate gamma and normal densities," Linear Algebra and Its Applications, vol. 396, no. 1-3, pp. 317-328, 2005.

[22] A. M. Mathai and H. J. Haubold, "On generalized distributions and pathways," Physics Letters A: General, Atomic and Solid State Physics, vol. 372, no. 12, pp. 2109-2113, 2008.

[23] H. J. Haubold and D. Kumar, "Fusion yield: Guderley model and Tsallis statistics," Journal of Plasma Physics, vol. 77, no. 1, pp. 114, 2011.

[24] D. Kumar and H. J. Haubold, "On extended thermonuclear functions through pathway model," Advances in Space Research, vol. 45, no. 5, pp. 698-708, 2010. 
[25] A. M. Mathai and R. K. Saxena, Generalized Hypergeometric Functions with Applications in Statistics and Physical Sciences, vol. 348 of Lecture Notes in Mathematics, Springer, New York, NY, USA, 1973.

[26] A. M. Mathai, A Handbook of Generalized Special Functions for Statistics and Physical Sciences, Clarendo Press, Oxford, UK, 1993.

[27] A. Erdélyi, Asymptotic Expansions, Dover, New York, NY, USA, 1956.

[28] F. W. J. Olver, Asymptotics and Spcecial Functions, Academic Press, New York, NY, USA, 1974.

[29] H. J. Haubold and A. M. Mathai, "Analytical results connecting stellar structure parameters and neutrino uxes," Annalen der Physik, vol. 44, no. 2, pp. 103-116, 1987. 

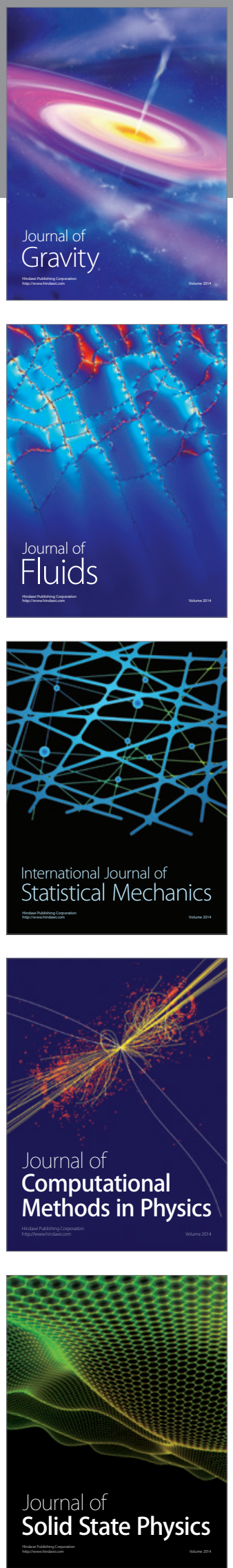

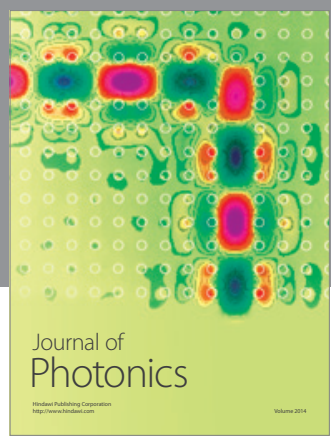

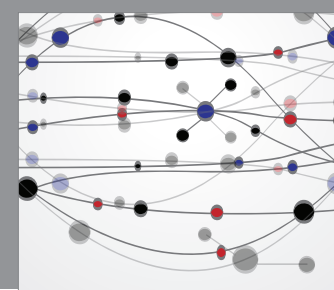

The Scientific World Journal

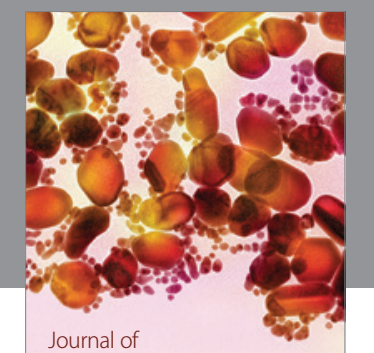

Soft Matter
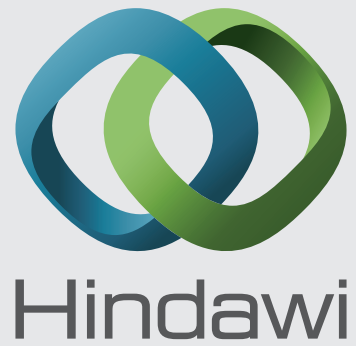

Submit your manuscripts at

http://www.hindawi.com
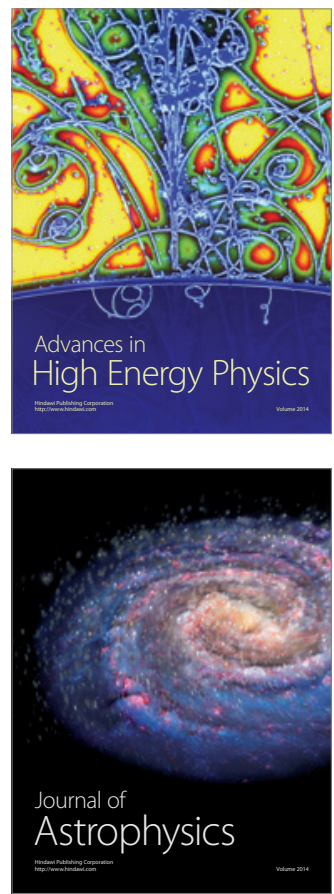
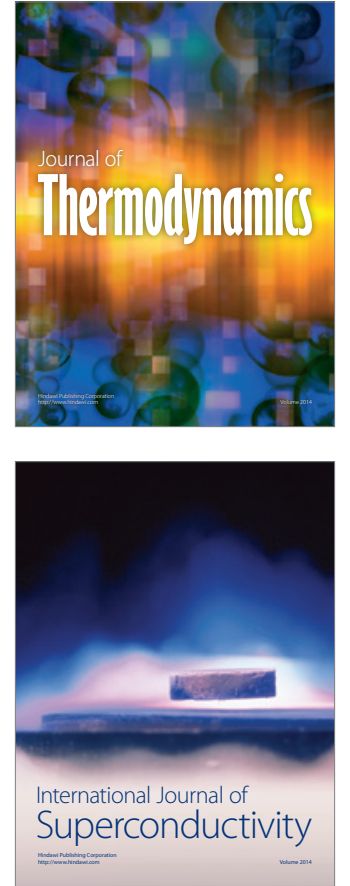
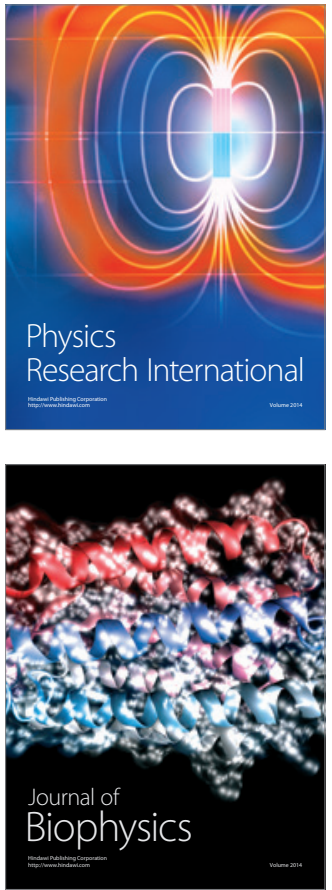
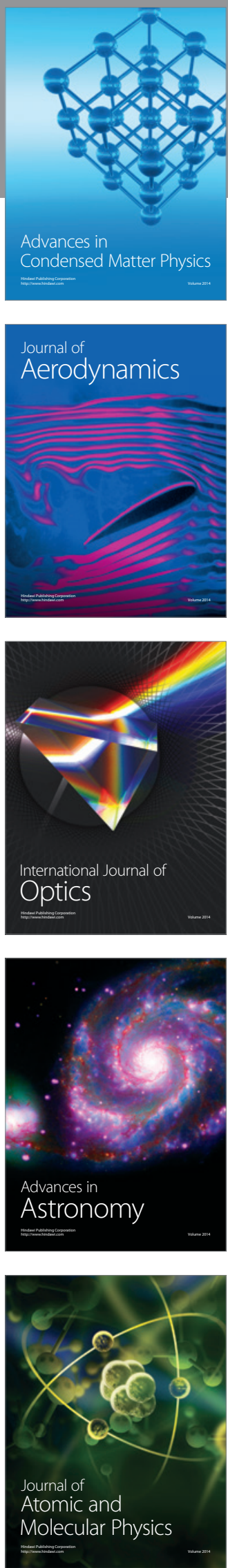